\title{
HER-2/neu Testing in Breast Carcinoma: A Combined Immunohistochemical and Fluorescence In Situ Hybridization Approach
}

Ren L. Ridolfi, M.D., Mehdi R. Jamehdor, M.D., Janet M. Arber, M.D.

Southern California Permanente Medical Group, Regional Reference Laboratories, Immunohistochemistry (RR), Genetics Testing Laboratory (MJ), North Hollywood, California, and Department of Pathology,

Baldwin Park Medical Center, Los Angeles, California (JA)

We evaluated 750 consecutive invasive breast carcinomas for HER-2/neu utilizing a combination of immunohistochemical (IHC) and fluorescence in situ hybridization (FISH) methodologies. IHC reactions of $3+$ were considered HER-2/neu positive and 0 and $1+$ IHC reactions were considered HER-2/neu negative. IHC reactions of $2+$ were considered inconclusive and reflexed to FISH analysis. In addition, a $10 \%$ sampling and validation FISH analysis was performed on the positive and negative IHC tests. One hundred thirty-eight cases (18.4\%) were HER-2/neu positive by IHC and/or FISH. One hundred twenty-three of the positive cases $(89 \%)$ were $3+$ IHC reactions and 14 positive cases were inconclusive by IHC and amplified by FISH. There was concordance with FISH in 77 of $78(98.7 \%)$ of the positive or negative IHC cases that were tested $(95 \%$ confidence interval $[\mathrm{CI}]=93.1$ to $100 \%)$. A single IHC-negative case showed HER-2/neu amplification by FISH. Thirty-nine cases were 2+ IHC (5.2\%); 14 (36\%) were amplified, 24 (62\%) were not amplified, and one was not interpretable. HER-2/neu positivity was observed in $34 \%$ of grade 3 ductal carcinomas, $11.4 \%$ of grade 2 ductal carcinomas, $3.2 \%$ of grade 1 ductal carcinomas, and $3.2 \%$ of lobular carcinomas. Occasional cases with discordant IHC expression of HER-2/neu within the in situ and invasive carcinoma elements were also identified. IHC reliably characterized HER-2/neu in approximately $95 \%$ of the cases studied $(95 \% \mathrm{CI}=\mathbf{9 3 . 0}$ to $96.2 \%$ ) and was effective as a primary method for evaluating HER$2 /$ neu status. In this study, $2+$ IHC reactions were a heterogeneous group best regarded as indeterminate or inconclusive; in this series, only $36 \%$ were

Copyright (C) 2000 by The United States and Canadian Academy of Pathology, Inc.

VOL. 13, NO. 8, P. 866, 2000 Printed in the U.S.A.

Date of acceptance: February 3, 2000.

Address reprint requests to: Ren L. Ridolfi, M.D., SCPMG Regional Reference Laboratories, Immunohistochemistry, 11668 Sherman Way, North Hollywood, CA 91605; e-mail: ren.l.ridolfi@kp.org; fax: 818-503-6714. amplified by FISH analysis. Our findings suggest that a combination of IHC and FISH testing with FISH analysis performed reflexly on all 2+ IHC cases can optimize HER-2/neu testing.

KEY WORDS: Breast carcinoma, FISH, HER-2/neu, Immunohistochemistry.

Mod Pathol 2000;13(8):866-873

At this time, the ideal method for accurately determining HER-2/ neu status in breast carcinoma has yet to be determined $(1,2)$. Both immunohistochemical (IHC) and fluorescence in situ hybridization (FISH) methodologies have been approved for clinical usage by the FDA. There are advantages and disadvantages to each methodology. IHC is accurate, relatively inexpensive, and can be performed quickly with little technical difficulty $(1,2)$. It is, however, not an optimal quantitative assay, and there is significant variation between different antibodies available for use (3-5). FISH testing is highly accurate and relatively easy to quantitate, but more expensive, time consuming, and technically difficult $(1,2)$. It has been suggested that perhaps a combination approach using both methodologies for HER-2/ neu testing might be advantageous (6).

Currently the DAKO HercepTest (DAKO, Carpinteria, CA) is the only IHC test approved by the FDA for evaluating HER-2/neu status in breast carcinoma (2). Although its overall reported performance has been good, there have been questions raised concerning its accuracy, particularly related to $2+$ positive reactions (7). Such reactions were originally recommended to be interpreted as "indeterminate" instead of "weakly positive" and did not correlate well with the clinical trial assay (CTA) investigational IHC test $(6,8)$. Because of these uncertainties (as well as cost considerations), we elected to use the DAKO polyclonal antibody (as used in the HercepTest) utilizing a standard HIER IHC technique to evaluate 750 breast carcinomas 
consecutively submitted for HER-2/ neu evaluation. The IHC assay was calibrated with the HercepTest and validated against an FDA-approved FISH test. IHC results were scored as with the HercepTest, but $2+$ IHC reactions were considered inconclusive instead of weakly positive and reflexed for further FISH testing. All $3+$ IHC positive and 0 to $1+$ negative IHC reactions were interpreted as such with validation FISH analysis performed on a subset of each group.

\section{MATERIALS AND METHODS}

\section{Case Selection and Specimen Processing}

All IHC and FISH testing was performed in Southern California Permanente Medical Group Regional Laboratories on breast carcinomas consecutively submitted for HER-2/neu status from all 11 Kaiser Medical Centers in southern California. These tissues were all fixed in a standard fashion at each medical center using $10 \%$ buffered formalin. All tissues were processed and paraffin-embedded at a single regional histology laboratory, except for one medical center, which processed tissues on site using a comparable technique. Length of fixation varied to some degree, but was generally between 12 and 24 hours.

\section{Immunohistochemistry Methodology}

Testing was performed on a Techmate 1000 (Ventana, Tucson, AZ). Tissue sectioned at $4 \mu \mathrm{m}$ was mounted on silanized capillary gap slides (Ventana), baked at $60^{\circ} \mathrm{C}$ for a minimum of $60 \mathrm{~min}$, deparaffinized with xylene, and rehydrated through graded alcohols to distilled water. Sections were then placed in 0.01-M citrate buffer at $\mathrm{pH} 6.0$ and heated in a steamer (Black \& Decker, Model H390) for $20 \mathrm{~min}$ at $90^{\circ} \mathrm{C}$. Anti-HER-2/ neu polyclonal antibody (DAKO) was used at a dilution of 1:2000. A secondary biotinylated antibody was used and reactivity detected by an avidin-biotin immunoperoxidase system employing 3'-diaminobenzidine tetrahydrochloride as the chromogen.

The IHC test was initially calibrated against cell button control slides from the DAKO HercepTest to yield negative, weak, and strong membrane staining on the appropriate cells. It was then parallel tested with the ONCOR HER-2/neu Gene Detection System (Ventana) on 20 cases yielding complete concordance with 0 to $1+$ (negative) and $3+$ (strongly positive) reactions and a single discordance in a 2+ IHC case.

\section{Immunohistochemistry Interpretation}

Membrane staining was scored 0 to $3+$, corresponding to results as described with the DAKO
HercepTest, with at least $10 \%$ of the cells staining for 1 to $3+$ reactions. Results were interpreted as negative with 0 and $1+$ reactions, positive with $3+$ reactions, and inconclusive with $2+$ reactions. Positive and negative controls were run with each case. The positive control always included a $3+$ reacting tumor and normal nonreacting ducts. All cases were interpreted by one of two pathologists (RR, JA), usually independently.

Cases interpreted as positive or negative for HER2/ neu overexpression by IHC methodology were reported as such. Cases that were inconclusive by IHC methodology were further tested by FISH methodology and reported based on the FISH findings (amplified or not amplified). In addition, approximately every 10th negative or positive case was also studied by FISH methodology as a validation procedure. All sections submitted for FISH analysis were screened by a pathologist to insure they contained appropriate tissue.

\section{FISH Methodology and Interpretation}

Four- $\mu \mathrm{m}$ thick sections of formalin-fixed paraffin-embedded tissue mounted on silanized slides were processed in the genetics laboratory for FISH study. An accompanying scored hematoxylin and eosin stained slide was received with each case. The slides were pretreated chemically and enzymatically to remove proteins that block DNA access. After denaturation, a hybridization solution containing labeled DNA probe complementary to the HER-2/neu gene sequence was applied to the tissue section, which was then incubated under conditions favorable for annealing of probe DNA and the genomic DNA sequence. After hybridization, the unannealed probe was washed off and the hybridized probe was detected using a fluorescently tagged ligand and a counterstain. An epifluorescence microscope (Zeiss Axioscope) equipped with an appropriate filter set was used to score the number of signals per nucleus according to the guidelines described by the manufacturer.

In 102 of a total of 117 cases, the ONCOR HER2/ neu Gene Detection System from Ventana Medical System, Inc. was used. In 15 cases, the PathVysion HER-2/ neu DNA Probe Kit from Vysis, Inc. was used. Scoring of signals was performed according to the manufacturer's guidelines. With the ONCOR probe, signals from 40 randomly selected cancer nuclei from two distinct areas were enumerated. A mean signal of greater than four indicated that HER-2/ neu amplification was identified, whereas a mean signal of less than or equal to four indicated that HER-2/ neu gene amplification was not identified. With the Vysis probe, signals from 60 cancer nuclei from two distinct areas of the tumor were enumerated and a ratio of the total number of 
HER-2/ neu signals to the total number of chromosome 17 signals (CEP 17) was calculated. A ratio equal to or greater than two indicated HER-2/neu gene amplification, whereas a ratio of less than two indicated HER-2/neu gene amplification was not identified.

\section{Grading}

A modified Bloom-Richardson grading scheme was used for grading all breast carcinomas (9). Lobular carcinomas included nuclear grade 1 and 2 tumors but not pleomorphic (nuclear grade 3 ) variants that were included among other subtypes.

\section{RESULTS}

Utilizing a combination of IHC and FISH results, we found that a total of 138 cases $(18.4 \%)$ of 750 exhibited HER-2/ neu overexpression or amplification and considered them to be HER-2/ neu positive (Table 1). Six hundred eleven cases were HER-2/ neu negative and one case was inconclusive.

By IHC alone, 123 cases were $3+$ positive $(16.4 \%)$, 39 cases were $2+$ inconclusive $(5.2 \%)$, and 588 cases were 0 to $1+$ negative $(78.4 \%)$. Within the positive cases, membrane staining was fairly uniform throughout and present in the majority of invasive tumor cells (Figs. 1, 2, 3, and 4). Some cases also showed nonspecific cytoplasmic staining.

\section{Inconclusive Cases (IHC)}

The 39 inconclusive cases displayed $2+$ membrane staining in at least $10 \%$ of the invasive tumor cells and usually had been repeated with similar results. Some cases were difficult to characterize as $2+$ (either $1+$ versus $2+$ or $2+$ versus $3+$ ).

Ten of the inconclusive cases were needle biopsies with a minority component of neoplastic cells showing $2+$ membrane staining, often associated with accentuated staining at the biopsy edges where the reaction appeared artifactual. These cases were interpreted as inconclusive in part because of limitations in confidently characterizing the reaction. Technical limitations such as uneven staining (probably related to suboptimal fixation) contributed to an inconclusive interpretation in

TABLE 1. Combined Immunohistochemical and FISH HER-2/neu Results

\begin{tabular}{lcc}
\hline & IHC Only & $\begin{array}{c}\text { Total } \\
\text { (IHC and/or FISH) }\end{array}$ \\
\hline Positive & $123(16.4 \%)$ & $138(18.4 \%)$ \\
Inconclusive & $39(5.2 \%)$ & $1(<1 \%)$ \\
Negative & $588(78.4 \%)$ & $611(81.5 \%)$ \\
Total & 750 & 750 \\
\hline
\end{tabular}

IHC, immunohistochemical; FISH, fluorescence in situ hybridization.

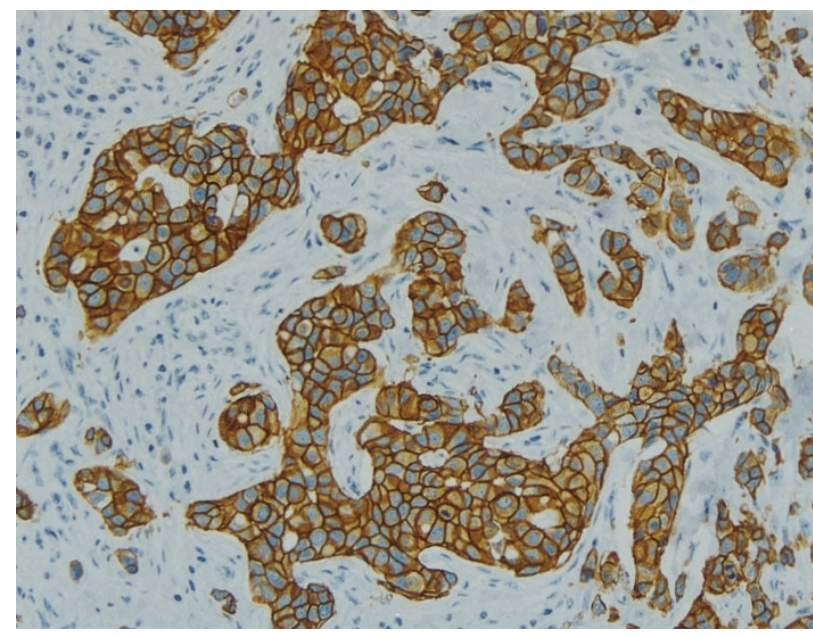

FIGURE 1. Invasive ductal carcinoma showing diffuse and uniform $3+$ IHC HER-2/ neu membrane staining (original magnification, 100×).

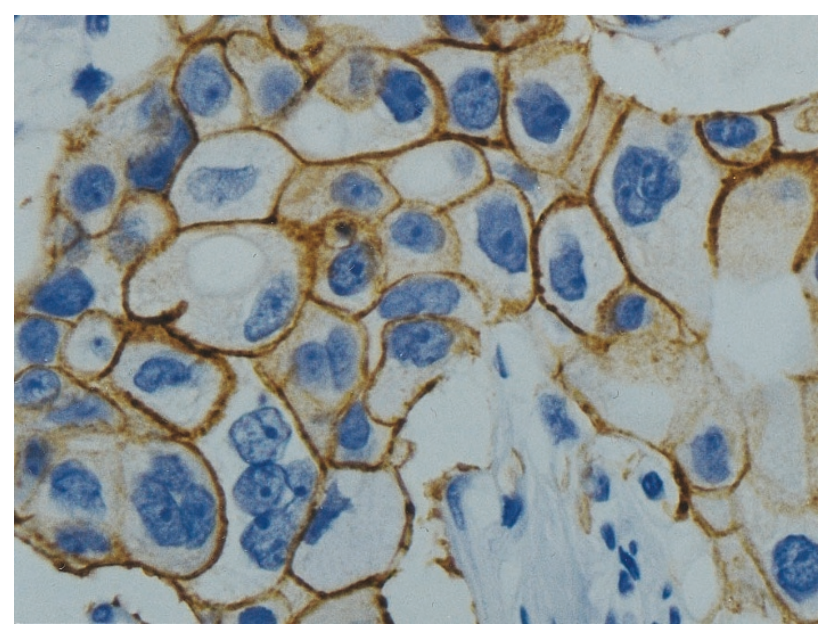

FIGURE 2. $3+$ IHC HER-2/ пеu membrane staining (original magnification, $400 \times)$.

some cases; however, these technical problems were also occasionally observed in $3+$ positive and negative cases.

\section{FISH Studies}

All $213+$ IHC positive cases on which validation FISH analysis was performed were amplified $(0 \%$ negative FISH cases with a $95 \%$ CI of 0 to $16 \%$; the overall CI is actually less because additional tests performed outside the study group have to date all been concordant with FISH results) (Table 2). Seventeen of the $213+$ IHC cases exhibited more than 20 signals per nucleus (Oncor) or a ratio of more than 10 (Vysis) (Fig. 5). The remaining four cases showed amplification ranging from 8.2 to 19 signals per nucleus. Among 57 cases interpreted negative by IHC, all but one showed no amplification by FISH analysis $(1.75 \%$ positive FISH cases with a $95 \%$ CI of 0.04 to $9.4 \%$ ). A single case showing $1+$ IHC membrane staining exhibited amplification by 


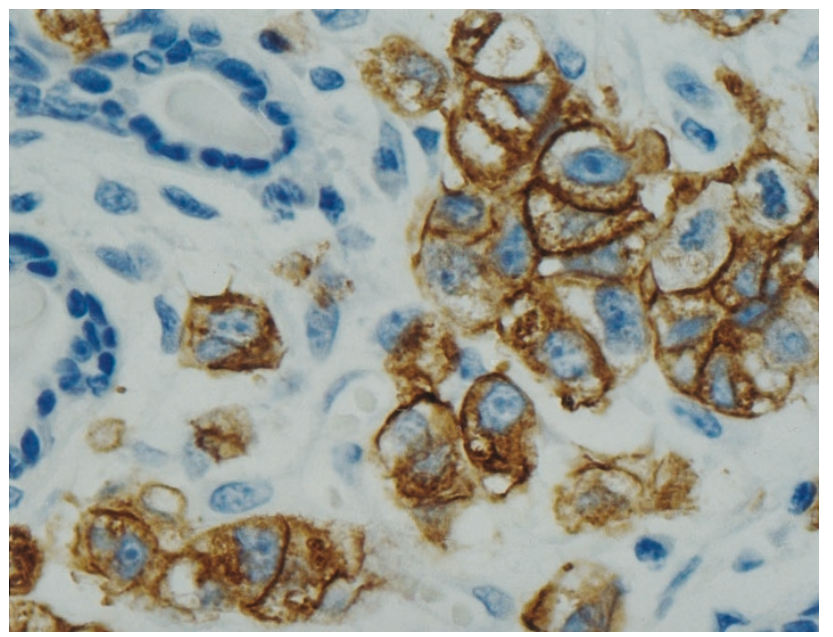

FIGURE 3. $3+$ IHC HER-2/neu membrane and nonspecific cytoplasmic staining with negative internal control normal mammary ducts (original magnification, $400 \times$ ).

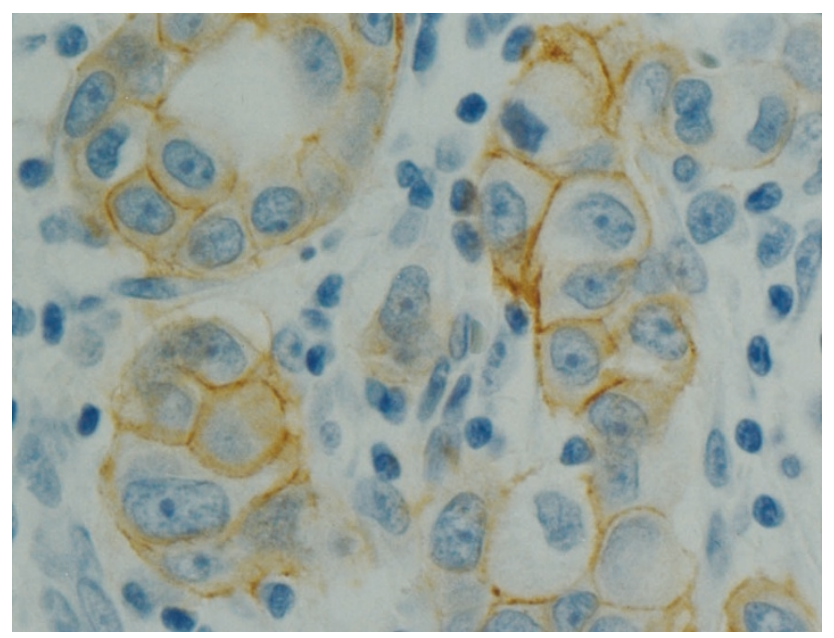

FIGURE 4. $2+$ IHC HER-2/neu membrane staining completely encircling most tumor cells (original magnification, $400 \times$ ).

TABLE 2. HER-2/neu FISH Studies

\begin{tabular}{cccc}
\hline IHC & FISH Positive & FISH Negative & Total \\
\hline $3+$ & 21 & 0 & 21 \\
$2+$ & 14 & 24 & $39^{*}$ \\
$0-1+$ & 1 & 56 & 57 \\
& & & 117 \\
\hline
\end{tabular}

* A single case was not interpretable by FISH.

IHC, immunohistochemical; FISH, fluorescence in situ hybridization.

FISH (15.5 signals per nucleus). Thus, there was concordance in 77 of 78 validation FISH studies (98.7\% with a $95 \%$ CI of 93.1 to $100 \%$ ).

Among the 39 cases interpreted 2+ IHC inconclusive, 14 (36\%) were amplified by FISH and 24 were not. One case could not be interpreted for technical reasons. The $2+$ IHC cases that were amplified by FISH showed a range of positivity from 4.1 to more than 20 signals per nucleus (Fig. 6). Four cases displayed between 4 to 10 signals per

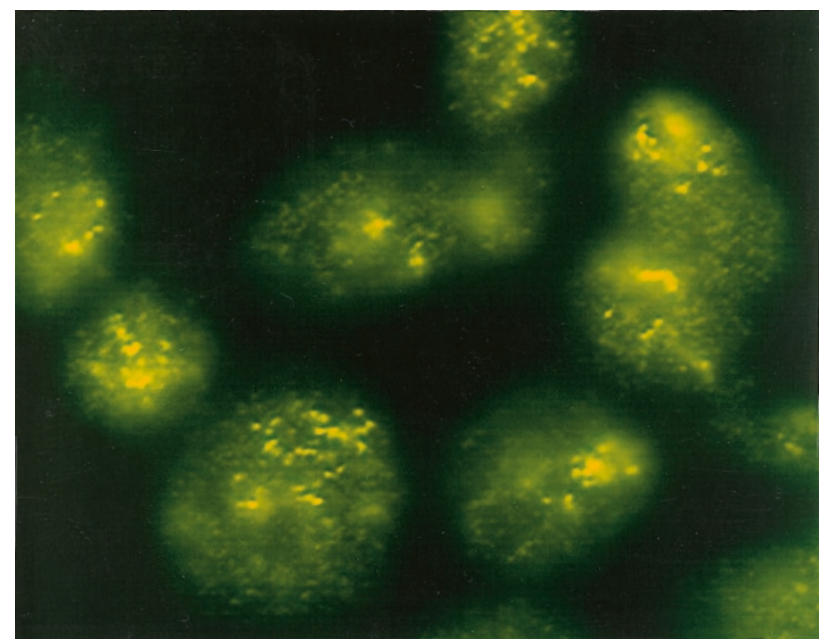

FIGURE 5. Invasive ductal carcinoma showing high HER-2/neu amplification by FISH (more than 20 signals per nucleus). Same case as seen in Figure 3.

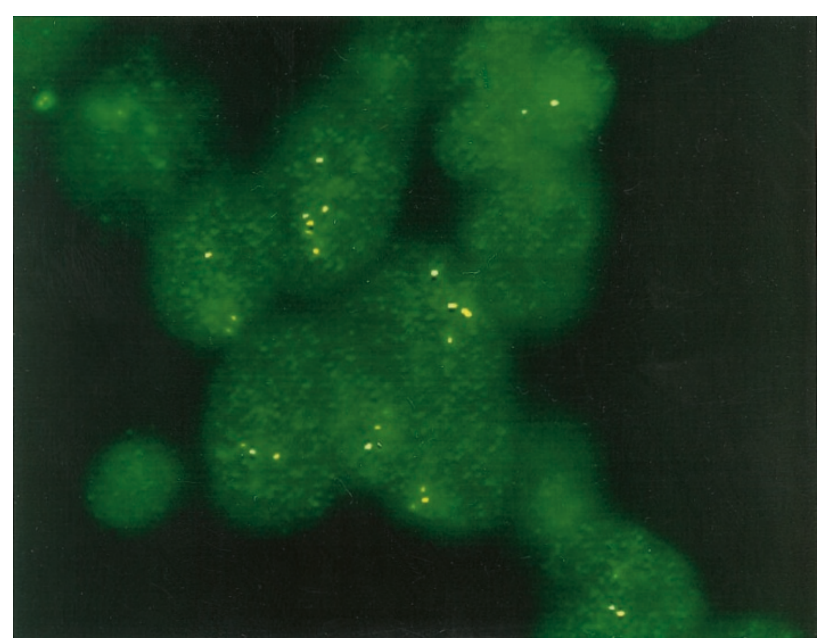

FIGURE 6. Invasive ductal carcinoma showing weak to moderate HER-2/ neu amplification by FISH (7.2 signals per nucleus). Same case as seen in Figure 4.

nucleus, five cases between 10.1 to 15 signals per nucleus, and five cases more than 15.1 signals per nucleus.

In four additional cases 2 to $3+$ IHC membrane staining was present in the ductal carcinoma in situ (DCIS) component of the tumor, whereas the invasive component was IHC negative (Fig. 7). We performed FISH analysis on these cases but did not include them with the 2+ IHC inconclusive cases. Two showed amplification by FISH within both the in situ and invasive components and two cases showed amplification within the DCIS elements but no amplification in the invasive elements. These were all interpreted as HER-2/neu negative in the final analysis. In all other cases with a mixture of DCIS and invasive carcinoma, the IHC reactions were similar or minimally different in both components. 


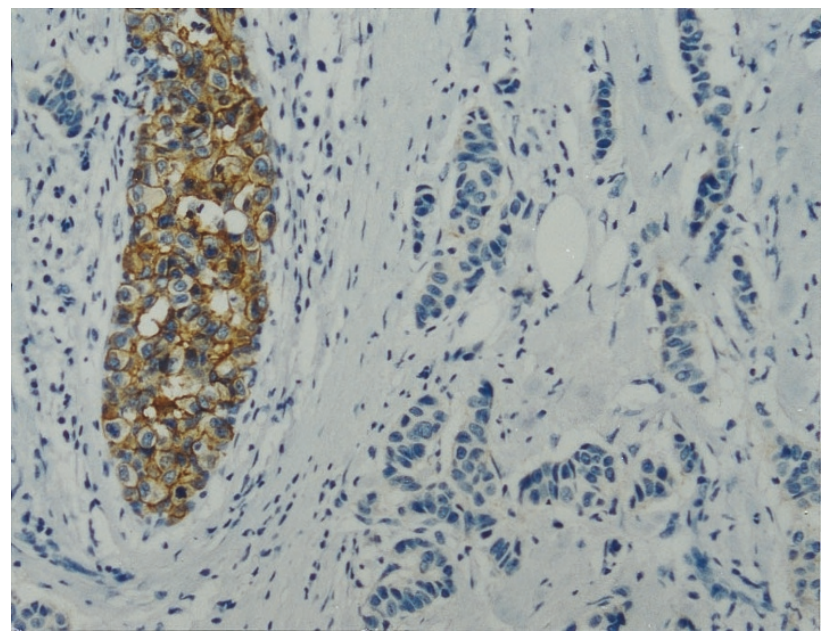

FIGURE 7. In situ and invasive ductal carcinoma showing discordant HER-2/neu protein expression. 3+ IHC membrane staining is noted within the DCIS (left) and negative staining within the invasive component to the right (original magnification, $100 \times$ ).

\section{Pathology}

One hundred three of 304 (34\%) grade 3 ductal carcinomas, 30 of $264(11.4 \%)$ grade 2 ductal carcinomas, and three of $93(3.2 \%)$ grade 1 ductal carcinomas were HER-2/ neu positive (Table 3). Two lobular carcinomas among 63 studied $(3.2 \%)$ were positive. All seven colloid carcinomas were HER-2/ neu negative as were 19 additional special subtype carcinomas including nine mixed ductal/lobular carcinomas.

\section{DISCUSSION}

The currently evolving therapeutic and prognostic implications of HER-2/ neu status in breast carcinoma have resulted in greater focus on HER-2/ neu testing. IHC on paraffin embedded tissue and FISH are the two methodologies currently FDAapproved for use in HER-2/ neu testing in selected circumstances and are generally available to most clinicians for such evaluation. Other methodologies, including protein blot analysis, IHC on frozen tissue, and ELISA are less practical for routine use $(1,2)$.

TABLE 3. HER-2/neu IHC/FISH: Pathology Correlations

\begin{tabular}{lrc}
\hline & Total & Positive \\
\hline Ductal-G1 & 93 & $3(3.2 \%)$ \\
Ductal-G2 & 264 & $30(11.4 \%)$ \\
Ductal-G3 & 304 & $103(34 \%)$ \\
Lobular & 63 & $2(3.2 \%)$ \\
Colloid & 7 & $0(0 \%)$ \\
Other & 19 & $0(0 \%)$ \\
\hline
\end{tabular}

With $\chi^{2}$ testing, the difference between G1, G2, and G3 are statistically significant $(P<.001$ overall with $\mathrm{G} 1$ versus $\mathrm{G} 2, P=.03$; $\mathrm{G} 2$ versus $\mathrm{G} 3, P<$ .001 ; and G1 versus G3, $P<.001$ ).

IHC, immunohistochemical; FISH, fluorescence in situ hybridization.
IHC is by far the most popular and accessible testing modality. It directly detects HER-2/neu protein overexpression and provides very accurate results with advantages including relative ease of performance, rapid turn-around time, and relatively low cost. In large part because of unavoidable variations in tissue fixation and processing, as well as variations in testing methodologies and subjectivity in grading, it is not an ideal quantitative assay and cases occur that are difficult to interpret. FISH methodology is more accurate and quantitatively more precise, but time consuming, technically demanding, and more expensive. Because it measures HER-2/neu gene amplification and not protein overexpression, FISH methodology theoretically may not be completely concordant with IHC results. Although there is good correlation between HER-2/ neu gene amplification and protein overexpression, approximately $3.5 \%$ of breast carcinomas overexpress HER-2/ neu without amplification and a small undetermined percentage amplify HER-2/ $n e u$ without overexpression (10-12). Direct comparisons between IHC on paraffin-embedded tissue and FISH HER-2/ neu testing are limited, but generally show high concordance $(13,14)$. Concordance has been stated to be particular high between IHC on frozen tissue and FISH, probably because fixation problems are obviated $(2,10)$. In several studies, the discordance has been in the range of 5 to $10 \%$ of the cases, and it is not clear if performing FISH in lieu of IHC is necessary or cost effective $(12,13)$. In addition, despite its clear superiority as a quantitative assay, clinically validated FISH reference values have not been established (15-18).

In the present study, we used a combination testing approach to evaluate HER-2/neu status in breast carcinomas, utilizing IHC as a primary testing modality and FISH in IHC inconclusive cases. Although we did not use the HercepTest kit, we used the same polyclonal DAKO antibody as provided in the kit with standard heat induced epitope retrieval. This methodology was calibrated with the HercepTest and validated against the FDAapproved ONCOR HER-2/neu FISH assay. We scored the IHC reactions as recommended for the HercepTest, but interpreted $2+$ membrane reactions as inconclusive instead of weakly positive.

Ninety-five per cent of the cases were interpretable by IHC alone and 5\% were considered inconclusive. IHC reliably identified all 3+ IHC HER-2/ neu overexpressors and all IHC negative cases ( 0 to $1+)$ with concordance with FISH findings in all but one case. One hundred twenty-three cases (16.4\%) were $3+$ overexpressors by IHC and another 39 cases $(5.2 \%)$ were characterized as $2+$ overexpressors and designated inconclusive in our study. Thus, 162 cases $(21.6 \%)$ were either $2+$ or $3+$ over- 
expressors by IHC. FISH analysis revealed 14 of 39 inconclusive $2+$ IHC cases $(36 \%)$ to be amplified utilizing current suggested reference values. In this study, the significance of a $2+$ IHC reaction was uncertain because the majority of such cases were not amplified by FISH. Although we suspect our results would be comparable using the HercepTest, we cannot state this with certainty and additional study of this issue would be of interest.

There is limited published information on $2+$ IHC reactions using the HercepTest. Forty-two per cent of $2+$ HercepTests were negative by the investigational IHC CTA used in the HERCEPTIN clinical trials (8). In a brief letter, Espinoza and Anguiano (19) reported $2+$ IHC reactions in $11.1 \%$ of their cases (162 of 1453) using the HercepTest, but did not give detailed FISH results on this subgroup. Among 21 of their positive HercepTests $(2+$ and $3+$ reactions), five (24\%) were not amplified by FISH, which we believe is cause for concern and is not acceptable. In this study, the $2+$ IHC cases were a heterogeneous group that was usually not amplified.

Nevertheless, we believe IHC may be reliably used as the primary methodology at this time for evaluating HER-2/ neu status. It directly measures HER-2/neu protein overexpression and reliably characterized $95 \%$ of the cases in this series in a cost-effective, expeditious fashion. The cost of the IHC test as performed in our laboratory is approximately $\$ 20$ versus $\$ 140$ for FISH analysis. Performing the HercepTest would cost about $\$ 50$ (excluding capital equipment expense for the DAKO Autostainer that is required to perform the test to FDA standards). The IHC methodology circumvents the potential small percentage of false negatives and false positives with FISH that are HER-2/neu amplified without overexpression or HER-2/ neu overexpressors that are not amplified. The only case in this series that was interpreted negative by IHC but was amplified by FISH was a grade 3 primary ductal carcinoma that displayed $1+$ membrane staining. Repeat IHC on the primary tumor and a lymph node metastasis showed similar $1+$ and focally $2+$ membrane staining. The significance of this isolated discordant result is not clear. It could represent a true false negative IHC or a HER-2/ neu amplified breast carcinoma without protein overexpression.

We also noted several additional unusual cases in which FISH analysis might be potentially misleading, especially if appropriate areas of a given slide are not evaluated. In this study, almost all cases with mixed DCIS/invasive elements exhibited concordant HER-2/neu IHC expression; however, in four cases the DCIS component showed 3+ membrane staining and the invasive component was negative. In two cases, the FISH findings were con- cordant with IHC; however, in the other two both the DCIS and invasive elements were amplified by FISH. Because HER-2/neu status is based on the findings within the invasive component, these cases might be considered positive based on the FISH findings if interpreted out of context. We believe, however, that the latter two cases may represent "false positive" FISH findings (amplified but not overexpressed) for several reasons. In each case, the IHC reaction showed clear $3+$ membrane staining within the DCIS component and no membrane reaction whatsoever in the invasive component. The discordance in these cases was unlikely due to fixation problems because vimentin staining in these areas confirmed the antigen integrity in both instances (20). It is also known that a higher percentage of DCIS cases (up to 55\% of high grade DCIS) are HER-2/ neu positive than invasive carcinomas (21). Furthermore, as noted above, a small percentage of invasive breast carcinomas demonstrate HER-2/ neu amplification but no detectable overexpression (11).

These two cases are unique in that a discordance in overexpression was apparent by IHC within the DCIS and invasive elements without a comparable discordance in amplification by FISH. We are not aware that this has been reported previously. IHC HER-2/ neu discordance between the DCIS and invasive components has been noted by some authors in at least nine previously reported cases; however, all were studied by IHC and none by FISH (22-24). These cases and several similar cases evaluated apart from this study series will be reported in more detail separately. Although very unusual, these cases exemplify a potential source of error in establishing accurate HER-2/neu status in cases with mixed DCIS/invasive elements. Unless areas of the tissue block are preselected carefully, FISH interpretation might be misleading and possibly yield false positive results.

There was a clearcut relationship between tumor grade and HER-2/ neu overexpression in this study (Table 3). HER-2/ neu positivity was present in 34\% of grade 3 ductal carcinomas, $11 \%$ of grade 2 ductal carcinomas, and only $3 \%$ of grade 1 ductal carcinomas. There were two positive lobular carcinomas among 63 tested (3\%) and no HER-2/neu overexpressors among seven colloid carcinomas. The reported positivity rate in relation to tumor grade and histologic subtype is extremely variable, much like the reported overall positivity rate in breast carcinoma in general $(1,2)$. Other studies have reported results similar to ours, but some have not (25-30). These variations are, in part, probably related to inconsistencies in categorizing and grading breast carcinomas and to the fact that some studies that have small numbers and selected populations of breast carcinoma patients. The current study pop- 
ulation includes an admixture of primary and recurrent breast carcinomas consecutively submitted for HER-2/neu study and thus is probably skewed with higher-grade tumors. The significant variation in HER-2/ neu positivity among different grade ductal carcinomas and lobular carcinomas may explain some of the variation reported in HER-2/neu positivity between different series with variations in case mix.

\section{Conclusion}

The current study found that a combined approach using both IHC and FISH methodologies can optimize HER-2/ neu testing on breast carcinomas. When properly calibrated, HER-2/ neu testing by IHC on paraffin-embedded tissue is a very good first choice methodology that, in this study, reliably characterized $95 \%$ of the cases, including all $3+$ positive and 0 to $1+$ negative cases. Approximately $5 \%$ of the cases were interpreted $2+$ by IHC (which we considered inconclusive) and were, with one exception, resolved by FISH analysis. The $2+$ IHC group in this study was heterogeneous in nature with only $36 \%$ showing HER-2/ neu gene amplification. Further studies of $2+$ IHC reactions utilizing the HercepTest in parallel with FISH analysis might be of value. There is also a need for establishing a clinically validated cut-off value for HER-2/neu FISH amplification against which IHC may be further compared and calibrated. This would potentially allow for more accurate and clinically meaningful HER-2/ neu testing in the future.

Acknowledgments: The authors thank Dr. Michael Kanter, M.D. for providing statistical analysis and Marlene Winger, H.T. for excellent technical support.

\section{REFERENCES}

1. Hanna W, Kahn HJ, Trudeau M. Evaluation of HER-2/neu (erbB-2) status in breast cancer: from bench to bedside. Mod Pathol 1999;12:827-34.

2. Ross JS, Fletcher JA. HER-2/neu (c-erb-B2) gene and protein in breast cancer. Am J Clin Pathol 1999;112(1 Suppl):553-67.

3. Press MF, Hung G, Godolphin W, Slamon DJ. Sensitivity of HER-2/neu antibodies in archival tissue samples: potential source of error in IHC studies of oncogene expression. Cancer Res 1994;54:2771-7.

4. Busmanis I, Feleppa F, Jones A, McGrath KM, Reed R, Collins $\mathrm{J}$, et al. Analysis of cerbB2 expression using a panel of 6 commercially available antibodies. Pathology 1994;26:261-7.

5. Bobrow CG, Happerfield LC, Millis RR. Comparison of immunological staining with different antibodies to the c-erbB-2 oncoprotein. Appl Immunohistochem 1996;4:12834.

6. Check W. More than one way to look for HER2. CAP Today 1999;13:48-52.

7. Roche PC, Ingle JN. Increased incidence of HER2 overexpression with FDA-approved antibody in breast cancer patients [letter]. J Clin Oncol 1999;17:434-5.
8. U S PMA. DAKO HercepTest [package insert]. Carpinteria, CA: DAKO; 1998.

9. Elston CW. Grading of invasive carcinoma of the breast. In: Page DL, Anderson TJ, editors. Diagnostic histopathology of the breast. Edinburgh: Churchill Livingstone; 1987. p. 30011.

10. Pauletti G, Godolphin W, Press MF, Slamon DJ. Detection and quantitation of HER-2/ neu gene amplification in human breast cancer archival material using fluorescence in situ hybridization. Oncogene 1996;13:63-72.

11. Hubbard AL, Doris CP, Thompson AM, Chetty U, Anderson TJ. Critical determination of the frequency of c-erbB-2 amplification in breast cancer. Br J Cancer 1994;70:434-9.

12. Uehara T, Kaneko Y, Kanda N, Yamamoto T, Higashi Y, Nomoto C, et al. C-erbB-2 and c-erbA-1 (ear-1) gene amplification and c-erbB-2 protein expression in Japanese breast cancers: their relationship to the histology and other disease parameters. Jpn J Cancer Res;81:620-4.

13. Couturier J, Nicolas A, Beuzeboc P, Vincent-Salomon A, Zafrani B. High correlation between erbB2 amplification detected by FISH and gene overexpression [abstract]. Mod Pathol 1999;12:18A.

14. Jacobs TW, Barnes MJ, Yaziji H, Gown AM, Scnitt SJ. Comparison of fluorescence in situ hybridization (FISH) and immunohistochemistry (IHC) for the evaluation of HER-2/ neu in breast cancer [abstract]. Mod Pathol 1999;12:23A.

15. Press MF, Pike MC, Chazin VR, Hung G, Udove JA, Markowicz M, et al. Her-2/ neu expression in node-negative breast cancer: direct tissue quantitation by computerized image analysis and association of overexpression with increased risk of recurrent disease. Cancer Res 1993;53:4960-70.

16. Pegram MD, Lipton A, Hayes DF, Weber BL, Baselga JM, Tripathy D, et al. Phase II study of receptor-enhanced chemosensitivity using recombinant humanized antip185HER2/ neu monoclonal antibody plus cisplatin in patients with HER2/neu-overexpressing metastatic breast cancer refractory to chemotherapy treatment. J Clin Oncol 1998;16:2659-71.

17. Muss HB, Thor AD, Berry DA, Kute T, Liu ET, Koerner F, et al. $\mathrm{C}$-erbB2 expression and response to adjuvant therapy in women with node positive early stage breast cancer. NEJM 1994;330:1260-6.

18. Thor AD, Berry DA, Budman DR. ErbB-2, p53 and efficacy of adjuvant therapy in lymph node positive breast cancer. J Natl Cancer Inst 1998;90:1346-60.

19. Espinosa F, Anguiano A. The HercepTest assay: another perspective [letter]. J Clin Oncol 1999;17:2293-4.

20. Battifora H. Assessment of antigen damage in immunohistochemistry: the vimentin internal control. Am J Clin Pathol 1991;96:669-71.

21. Lodato RF, Maguire HC, Greene MI, Weiner DB, Livolsi VA. IHC evaluation of c-erbB-2 oncogene expression in DCIS and ADH of the breast. Mod Pathol 1990;3:449-54.

22. Battifora H, Gaffey M, Esteban J, Mehta P, Bailey A, Faucett $\mathrm{C}$, et al. IHC assay of neu/c-erbB-2 oncogene product in paraffin-embedded tissue in early breast cancer: retrospective follow-up study of 245 stage I and II cases. Mod Pathol 1991;4:466-74.

23. Allred CA, Clark GM, Tandon AK, Molina R, Tormey DC, Osborne CK, et al. HER-2/ neu in node negative breast cancer: prognostic significance of overexpression influenced by the presence of in situ carcinoma. J Clin Oncol 1992;10:599605.

24. Tsuda H, Hirohashi S. Multiple developmental pathways of highly aggressive breast cancers disclosed by comparison of histological grades and c-erbB-2 expression patterns in both the non-invasive and invasive portions. Pathol Int 1998;48: 518-25.

25. Gusterson BA, Gelber RD, Goldhirsch A, Price KN, Save- 
Soderborgh, J, Anbazhagan R, et al. Prognostic importance of c-erbB-2 expression in breast cancer. J Clin Oncol 1992; 10:1049-56.

26. Toikkanen S, Helin H, Isola J, Joensuu H. Prognostic significance of HER-2 oncoprotein expression in breast cancer: a 30-year follow-up. J Clin Oncol 1992;10:1044-8.

27. Rozan S, Vincent-Salomon A, Zafrani B, Validire P, De Cremoux $\mathrm{P}$, Bernoux A, et al. No significant predictive value of c-erbB-2 or p53 expression regarding sensitivity to primary chemotherapy or radiotherapy in breast cancer. Int J Cancer 1998;79:27-33.
28. Porter PL, Garcia R, Moe R, Corwin DJ, Gown AM. C-erbB-2 oncogene protein in in situ and invasive lobular breast neoplasia. Cancer 1991;68:331-4.

29. Press MF, Bernstein L, Thomas PA, Meisner LF, Zhou JY, Ma Y, et al. HER-2/ neu gene amplification characterized by fluorescence in situ hybridization: poor prognosis in node negative breast carcinomas. J Clin Oncol 1997;15:2894-904.

30. Sjogren S, Inganis $\mathrm{M}$, Lindgren A, Holmberg L, Bergh J. Prognostic predictive value of c-erbB-2 overexpression in primary breast cancer, alone and in combination with other prognostic markers. J Clin Oncol 1998;16:462-9.

\section{Book Review}

\section{Mark HFL, editor: Medical Cytogenetics, 680 pp, New York, Marcel Dekker, 2000 (\$195.00).}

This compendium of cytogenetics is primarily aimed at the 550 or so physicians, Ph.D.s, clinical molecular biologists, and genetic counselors planning to take the certifying examination given by the 24th primary specialty board of the American Board of Medical Specialties. It goes without saying that I have picked up these facts from Dr Mark's book, which also contains everything else one might need if studying for the American Board of Medical Genetics. The book, however, will also be useful for residents in pathology, pediatrics, or internal medicine and for all others looking for a comprehensive coverage of this relatively young medical specialty.

The book consists of 20 chapters dealing with laboratory and clinical aspects of cytogenetics. Basic aspects of cytogenetics are systematically discussed, beginning with nomenclature, the nature of chromosomal and genetic abnormalities commonly encountered in practice, and current techniques used in the laboratories. Clinical problems that need cytogenetic work-up are presented in detail, and some are illustrated in black and white or color photographs. There are glossaries for readers less versed in cytogenetics. At the end, there is a chapter on standards for setting up a cytogenetics laboratory.

For those who do not know offhand the meaning of acronyms such as FISH, SKY, CGH, etc., this book is a godsend that should be on the reference shelf of their medical libraries. For those who want to use it as a textbook, the editor and his associates have prepared a set of study questions (included on the last 15 pages of the book). Professional cytogeneticists should have it handy for visitors or residents who are assigned to their laboratories for rotation. This is a truly multifunctional book that deserves to be made available to a wide range of readers.

\section{Ivan Damjanov \\ University of Kansas School of Medicine Kansas City, Kansas}

